\title{
Design and Construction of an Audio Surveillance System
}

\author{
I. A. Oguche \\ Main Author \\ Elect. \& Elect. Engineering \\ College of Engineering \\ Univ. of Agric.,Makurdi, Nigeria
}

\author{
J. U. Agber \\ Main Supervisor \\ Elect. \& Elect. Engineering \\ College of Engineering \\ Univ. of Agric.,Makurdi, Nigeria
}

\author{
N. S.Tarkaa \\ Minor Supervisor \\ Elect. \& Elect. Engineering \\ College of Engineering \\ Univ. of Agric.,Makurdi, Nigeria
}

\begin{abstract}
Surveillance is the monitoring of the behavior, activities, or other changing information, usually of people and events for the purpose of influencing, managing, directing, or protecting them. In this paper, an audio surveillance system was designed, simulated and constructed by replacing discrete components with an integrated circuit(CD2003) with the view of taking advantage of electret microphone ability to capture sound of low frequency and transmit it to the receiver where the signal can be retrieved. The audio signal was then amplified using two bipolar junction transistors (BJT), modulated and transmitted via an antenna. A provision for recording of the retrieved signal was made using a telephone set for continual logs of information for retrieval purposes at the receiver section. The circuit was designed and fully implemented with a designed output power of 0.3 watts which transmitted within 8-12meters radius and waveforms from proteus 8 professional and multism software were obtained and compared for performance and evaluation. This short range of coverage was designed and implemented with the aim of avoiding major interference from the surrounding since the system is meant to survey a small area like banking hall, bulk cash rooms and other sensitive areas.
\end{abstract}

\section{Keywords}

Electret, microphone, surveillance, antenna, waveform, monitoring, retrieved

\section{INTRODUCTION}

Surveillance is defined as covert observations of places and persons for the purpose of obtaining information [1]. The term covert infers that the operative conducting the surveillance is discrete and secretive. Surveillance that maintains a concealed, hidden, undetected nature clearly has the greatest chance of success because the subject of the surveillance will act or perform naturally. Remaining undetected during covert surveillance work often involves physical fatigue, mental stress, and very challenging situations. Currently, audio signals are making their way to public surveillance. For example, microphones are installed in locations with high crime rate by street gangs [2]. The advantage of audio is that the sensors are quite small and can be installed practically anywhere. Their low power-consumption allows local powersupply including wireless data transfer.

As population increases, the challenge in the rise in social vices also increases. The increase in crime in the present time has necessitated the need for security operatives, science and technology to indulge in research works towards finding solutions to combat this menace. In most cases, security operatives lack concrete evidences for prosecuting a potential criminal and this potential criminal go scot free. There are times or situations where verbal evidences are provided in court of law to evict or convict a suspect. In view of all this, audio surveillance system is important and necessary.This audio surveillance system employs the transmitting of audio signal or information from some particular points to a single receiver point. The principle of telecommunication, which the audio surveillance system is based on, uses a transmitter that generates or emits a particular radio wave frequency, which is used as the carrier of this audio signal. The transmitter electronically encapsulates the audio signal, magnifies and transmits it at a particular frequency via an antenna. The transmitted signal is received from the central reception point within the coverage distance of the transmitter. The received radio wave is then filtered, detected (demodulated) and converted back to audio and is heard on the loud speaker.

The history of audio surveillance involves four major aspects, the understanding of the science of sound, the evolution of technologies to project sound over distance, the evolution of devices to record sound, and laws to regulate the use of these technologies. Much of the current legislation regarding communications, recording, and wiretapping has its roots deep in the 19th century. Humans have been recording events for at least 2,000years, through images and later through text, but the recording of sound is a surprisingly recent event [3].

\section{LITERATURE REVIEW}

Surveillance has received a great attention in extreme active application-oriented research areas in computer vision, artificial intelligence, and image processing. Audio surveillance is conducted over both wired and wireless systems. The main focus in this paper is on wireless systems because audio communications are converted into radio-wave frequencies before transmission. The early use of monitoring system was the tube camera that was deployed to broadcast and monitor the industrial processing in the 1930s and 1940s [4], [5]. The traditional video surveillance systems - normally called Close-Circuit Television (CCTV) - was defective and costly since they were deployed by security teams to observe events in the scenes via visual display.

In recent decades, expansion in surveillance systems led to expansion in various prominent domains of technology and science such as homeland security, crime prevention through indoor and outdoor monitoring, elder care, accident detection, traffic monitoring, controlling and traffic flow analysis, 
airborne traffic management, maritime traffic control, counting moving object - pedestrians, vehicles, human behavior understanding, motion detection, activity analysis, identification, tracking and classification of vehicles, peoples, and any object of interest [5] - [21]. Encryption and acoustic surveillance are closely related, since encryption is one of the principal ways in which the privacy of communications is safeguarded. For this reason, acoustic surveillance, computer encryption, and cryptology share a common history in many respects [22].

In this paper the receiver section of the circuit has an RF amplifier which is a simple common emitter amplifier using a BF199, this is followed by the product detector using three times BF199 transistors. The audio from the product detector is passed through a simple low pass filter to the audio preamplifier using a BC549C transistor, then on to the audio amplifier using an LM386.

Work on an input amplifier for a FM-radio receiver with RF selection (88-108 $\mathrm{MHz}$ ) has been designed in the radio section[25]. It has about $25 \mathrm{~dB}$ gains in the frequency range 88-108 MHz. Mirror frequency rejection is between $5 \mathrm{~dB}$ to 9 $\mathrm{dB}$. Noise figure is about $7-10 \mathrm{~dB}$ at resonant frequency. The amplifier works fairly well, when it is connected to the rest of circuits to receive FM broadcast signals. The limitation of this is work is the inability to effectively control noise range due to high attenuation rate.A work on designing a short range wireless USB-FM transmitter which can transmit data contained in the USB flash drive over short distance using ROHM ${ }^{\circledR}$ BU9458kv Semiconductor chip was done [26]. The project has actually been done in two parts, designing and analyzing. The first section is FM Transmission and the second is USB-mp3 module. The FM Transmitter is designed by using two BC-547 transistors. The USB mp3- Module is designed using ROHM® BU9458kv Semiconductor chip. The $\mathrm{mp} 3$ songs can be played directly from the USB flash drive by using MODE-1(default mode) of the semiconductor chip without programming it. Successful Transmission of audio signal through the designed FM Transmitter is a proof of the correctness of the design. The experimental results on FM transmitter ensurethat the design is capable of transmitting USB data if it is implemented completely on commercial basis. The limitation of this system is the inability to transmit on a longer distance and much distortion on the transmitted data.

A walkie-talkie is a hand-held portable, bi-directional radio transceiver. Major characteristics include a half-duplex channel (only one radio transmits at a time, though any number can listen) and a push-to-talk switch that starts transmission. Hand-held transceivers became valuable communication tools for police, emergency services, and industrial and commercial users, and are also popular with some amateur radio operators.The personal walkie-talkie has now become popular again with the new U.S. Family Radio Service and similar unlicensed services in other countries. While FRS walkie-talkies are also sometimes used as toys because mass-production makes them low cost, they have proper super heterodyne receivers and are a useful communication tool for both business and personal use. Although this system works effectively, but it cannot record transmitted information directly due to the way it is designed [23], [24].

This research work of design and construction of audio surveillance system is based on the principles of a walkietalkie which comprises a transmitter section and a receiver section. Here, a recording device to cater for the recording of the received FM signal. Hence, the recorded information can then be retrieved for analysis and as evidence which is the main core aim of this paper (audio surveillance system). The transmitter section comprises the collection of audio sound, magnifying it and transmitting it at a design frequency of between $88-107 \mathrm{MHZ}$ via antenna, while the receiver section comprises the frequency selectors, filtering devices and decoding/detection of the collected audio signal before recording and outputting the signal via speaker. In this paper, Bipolar Junction Transistors (BJTs) are used in the design of both the transmitter and receiver circuits but an integrated circuit (IC) is used in the receiver sections to replace the assemblage of several discrete components which helped significantly to reduce loss of signals, noise, high attenuation rate and economically elimination of cost of replacing damaged components due to assemblage and testing.The chosen frequency is $100 \mathrm{MHz}$ and the transmitter power is 0.3 Watts to transmit at a distance of about $8-12 \mathrm{~m}$ radius to avoid major interference with other nearby systems and the mixer type used here is double-balanced mixer(DBM) for a fairly good output

\section{METHODOLOGY}

Fig 1 shows the block diagram of the transmitter section. It comprises the input block, which is basically a microphone, an amplifier block and a converter/modulator block, to which an antenna is attached. From the input, the signal is fed into the amplifier section but before amplification, at TP1 from the diagram, the signal from the microphone was tested, measured and record for comparative analysis and the same method was deplored at respectively to measure the amount of amplification that had taken place before conversion, modulation of the signal and transmission via the antenna.

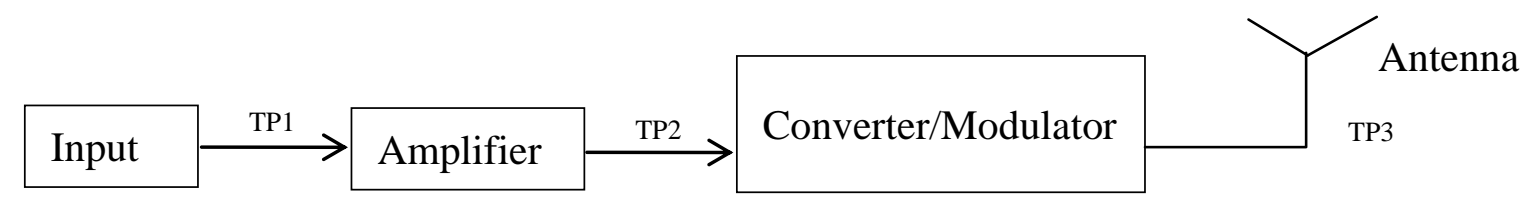

Fig. 1: The block diagram of the transmitter

Fig. 2 shows the FM transmitter circuit diagram. It is made up of the following main components: Oscillators, Microphones, Modulators and Amplifiers among other discrete components.
It is the circuitry representation of the block diagram above showing the two stages of amplification before feeding it into the LC tank and then transmission via the antenna 


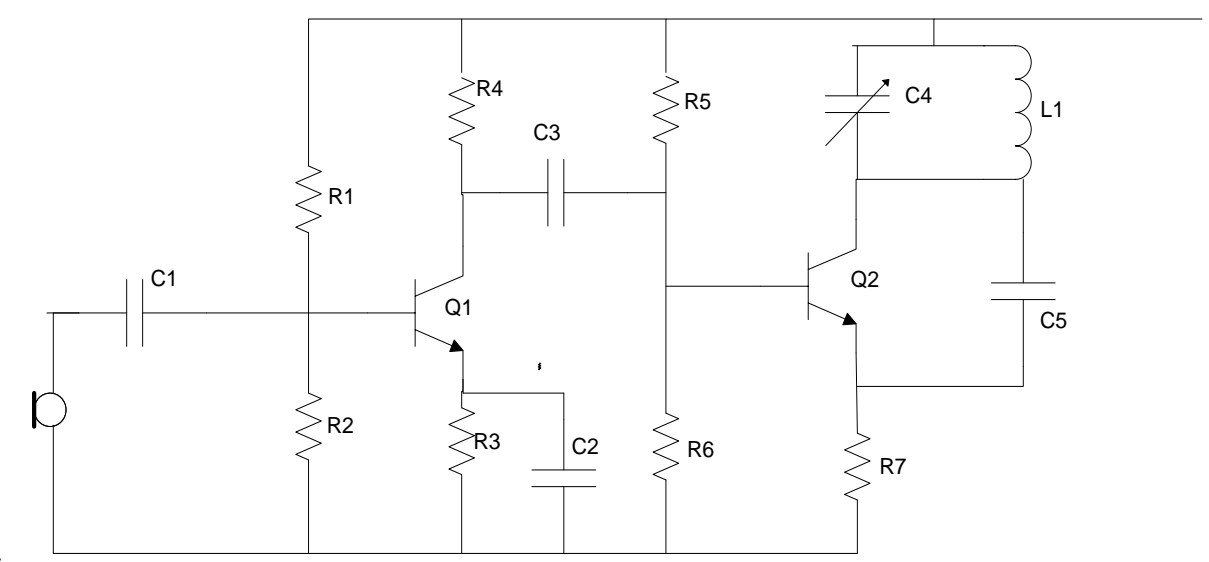

Fig. 2: Transmitter Circuit Diagram

$$
V_{B}=\frac{V_{c c} R_{2}}{R_{1}+R_{2}}
$$

$$
R_{B}=R_{1} \| R_{2}
$$

Taking Base-emitter loop, KVL yields

$V_{B}=I_{B} R_{B}+V_{B E}+I_{E} R_{3}$

$=\frac{I_{c}}{\beta_{f}} R_{B}+V_{B E}+\left(\frac{\beta_{f}+1}{\beta_{f}}\right) R_{3}$

$\frac{I_{c}}{\beta_{f}}\left[R_{B}+\left(\beta_{f}+1\right) R_{3}\right]+V_{B E}$

Note that every parameter in eqn. 3 is already known except $R_{B}$ which can be calculated. Eqn. (1) and (2) are solved simultaneously to obtain $\mathrm{R}_{1}$ and $\mathrm{R}_{2}$

\section{Assuming derived operation points:}

1. $\quad$ selecting $R_{3}$ to make the voltage drop $/ I_{E} R_{3} /$ about 1 volts for stability

2. $R_{3}$ about $10 R_{3}$. for transistors with $\beta_{f} \approx 100$, this choice suppresses $\beta_{f}$ variation by a fraction of about 10 compared to a design with $R_{3}$ equal to zero. But still allows $R_{B}$ to be large enough to prevent excessive shorting of the input signal to ground.

$$
V_{B}=\frac{V_{C C} R_{2}}{R_{1}+R_{2}} \text { and } R_{B}=\frac{R_{1 R_{2}}}{R_{1}+R_{2}}
$$

We can deduce that:
$R_{1}=\frac{V_{C C R_{B}}}{V_{B B}}$ and $R_{2}=\frac{V_{C C} R_{B}}{V_{C C}-V_{B B}}=\frac{R_{1} V_{B B}}{V_{C C}-V_{B B}}$

$V_{C C}=9 \mathrm{~V}, V_{C E}=4.5, I_{C}=1 \mathrm{~mA}$ and $V_{B E}=0.7$

$\therefore R_{3}=\frac{V}{I_{C}}=\frac{1}{1}=1 \mathrm{Kohms}$

$R_{B}=10 R_{E}=10 \mathrm{~K} \Omega$

$\therefore V_{B B}=\frac{1}{100}(10 \mathrm{~K}+101 * 1 \mathrm{~K})+0.7$

$=1.8 \mathrm{~V}$

$R_{1}=\frac{9 * 10}{1.8}=50 \mathrm{ohms}$

$R_{1}=\frac{9 * 10}{9-1.8}=12.5 \mathrm{ohms}$

Taking loop along the collector,

$V_{c c}=I_{c} R_{c}+V_{C E}+I_{E} R_{3}$

$R_{C}=\frac{V_{C C}-V_{C E-} I_{E} R_{3}}{I_{C}}$

$=9-0.3-1=\frac{9-4.5-1}{1}$

$=3.5 \mathrm{~K} \Omega$

\subsection{Design of FM Receiver Circuit}

Fig. 3 represents the receiver circuit diagram for effective operation of the audio surveillance system after calculating the corresponding values of the designed components 


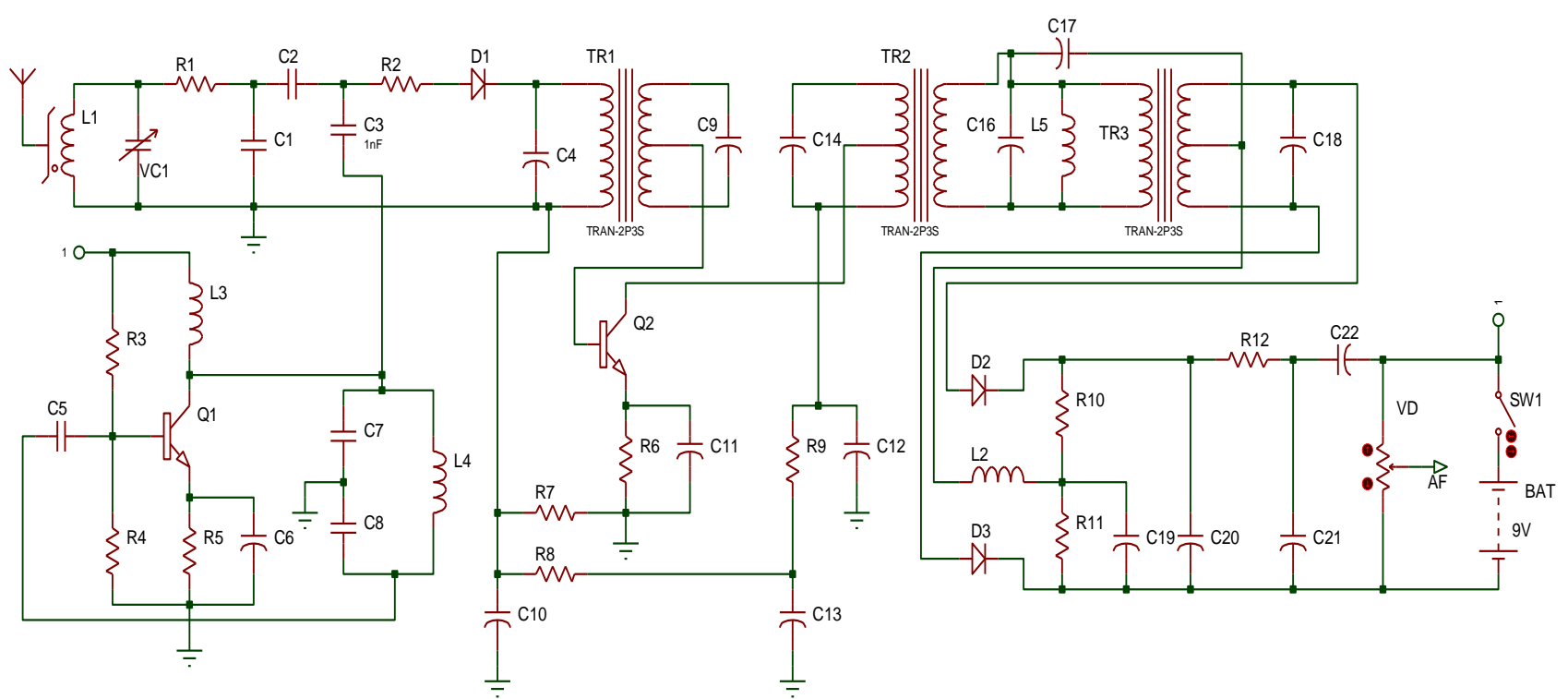

Fig. 3: Schematic diagram of FM 100MHz receiver

\section{SIMULATION RESULTS OF TRANSMITTER CIRCUIT USING PROTEUS IDE}

Figure 2 represents the circuit diagram of the transmitter to be simulated. Figure 4.1 shows the simulated output of the circuit using wave sound. Figure 4.2 shows the simulated output of the circuit using sinusoidal wave while figure 4.3 shows the simulated output of the Colpitt's Oscilator circuit.

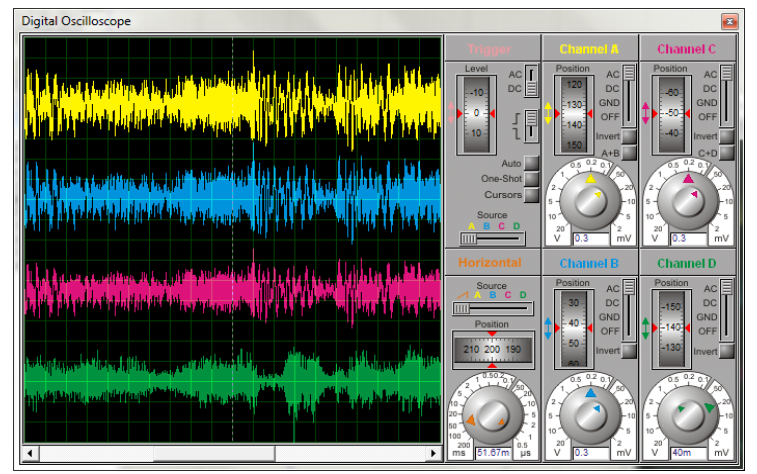

Fig. 4.1: Simulation of designed Transmitter using WAV sound

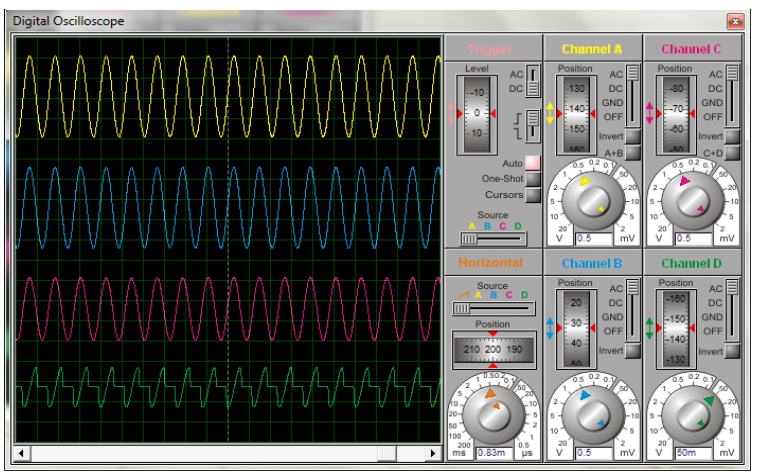

Fig. 4.2: Simulation of designed Transmitter using sinusoidal wave

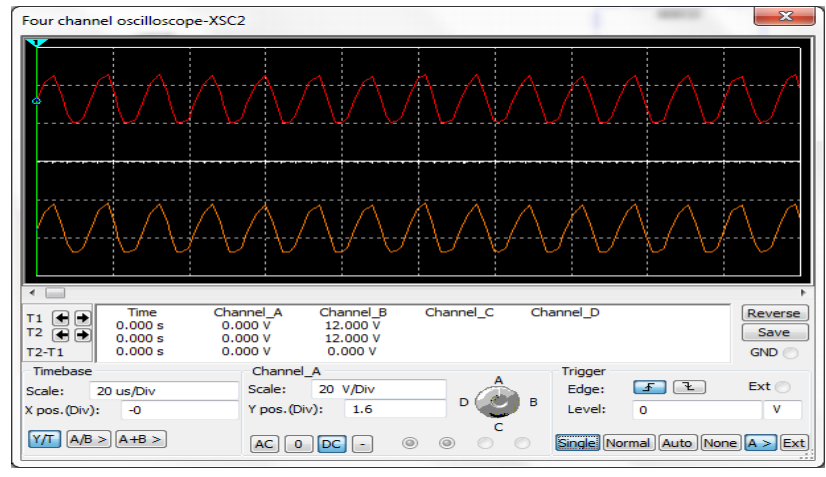

Fig. 4.3: Colpitt's oscillator waveform

\subsection{Results from Circuit Testing}

This section shows the results of the tests carried out on the transmitter and the receiver circuits using Digital Oscilloscope to view the various responses of the signal after being transmitted and fed into the receiver and their corresponding displays are as shown in figures $4.3-4.10$ respectively.

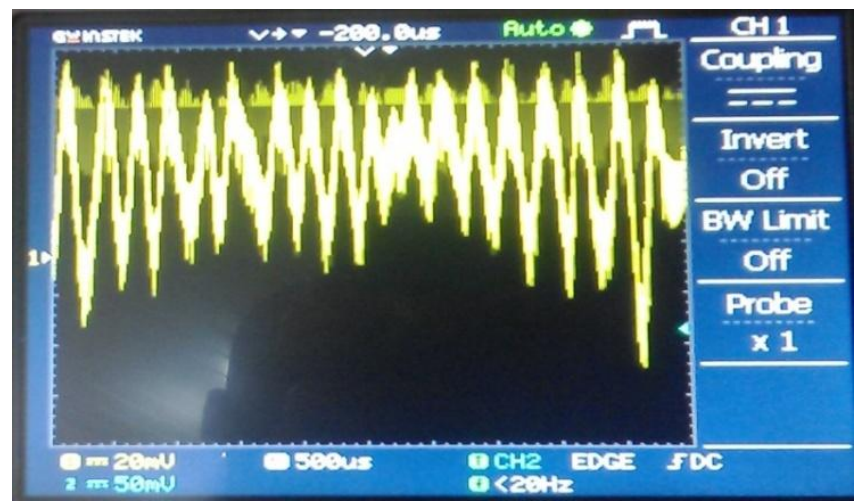

Figure 4.4: The waveform of the input signal to the base of the first transistor of the transmitter circuit when fed with audio signal. 


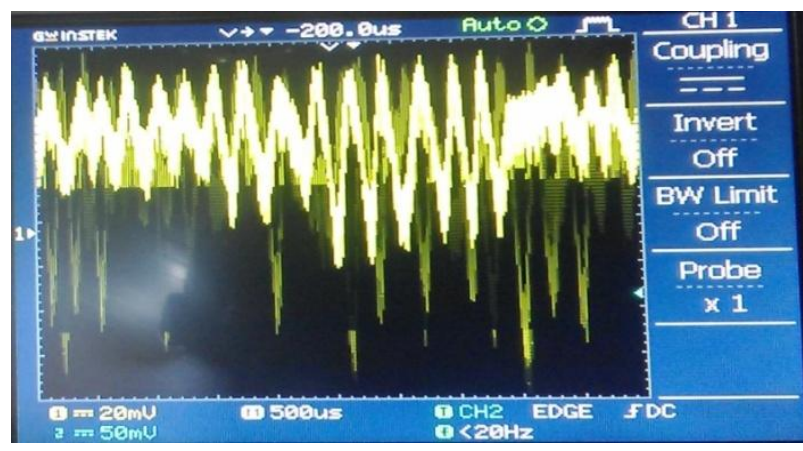

Figure 4.5: The waveform of the input signal to the base of the second transistor of the transmitter circuit when fed with audio signal

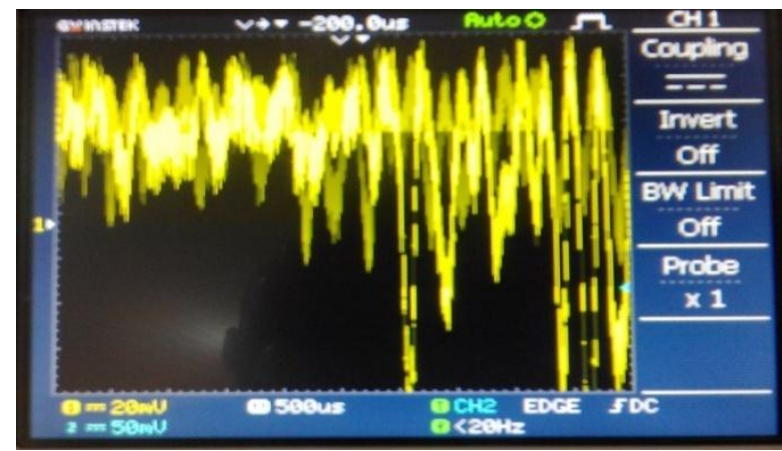

Figure 4.6: The waveform of the output signal to the LC tank terminal of the transmitter circuit when fed with audio signal.

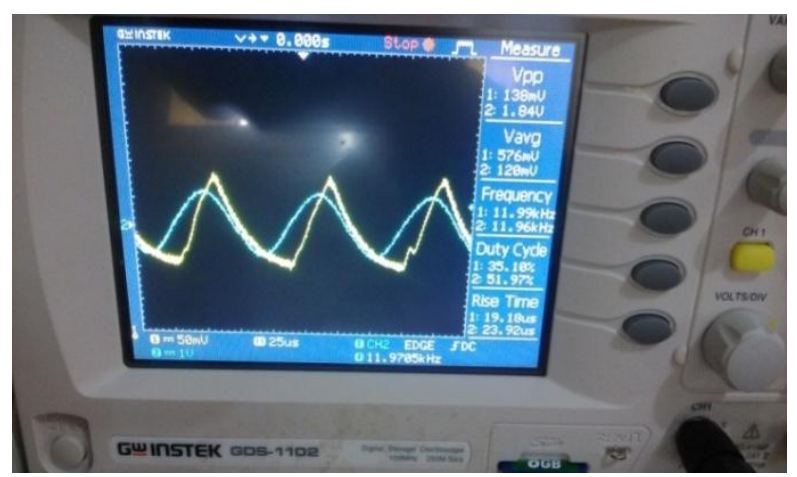

Figure 4.7: Output of the receiver circuit (at speaker terminal

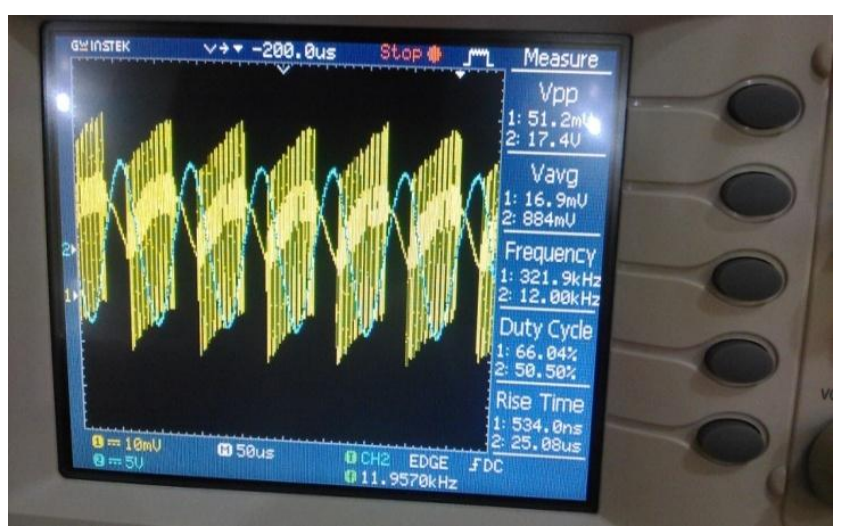

Figure 4.8: Transmitted signal(oscillator output)

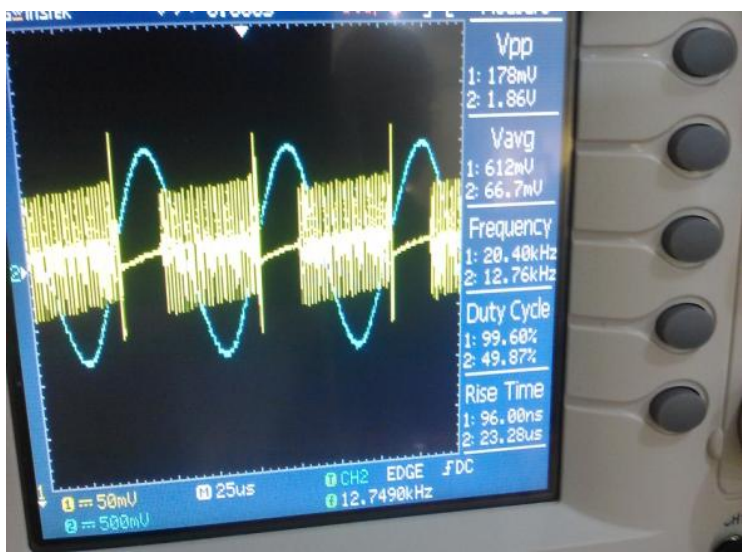

Figure 4.9: Received signal (at TP1 of RADIO)

\section{CONSTRUCTION}

In constructing the circuit, the following steps were taken:

1. It was ensured that the components were assembled externally to confirm the functionality of the various components together before transferring them on the vero board

2. It was equally ensured that every confirmed damaged or faulty components were replaced.

3. It was also ensured that all flaws on the vero board were removed to avoid bridging before powering testing the circuit

The constructed circuit was packaged. Packaging helps to provide:

i. Physical protection for the often sensitive components like ICs which are vulnerable to static charges.

ii. It enhances the beauty of the project.

iii. It reduces electrical shocks that may arise from contact with parts of the circuit that may have live voltages.

In this packaging, plastic is used for the encasement of this project due to its insulation property. Weight, compatibility and efficiency of the components are some of the factors considered in the construction of this project. The finished product of the transmitter is shown in Plate 3.1 and the receiver shown in Plate 3.2. The combination of the two, which forms the entire system is shown in Plate 3.3.

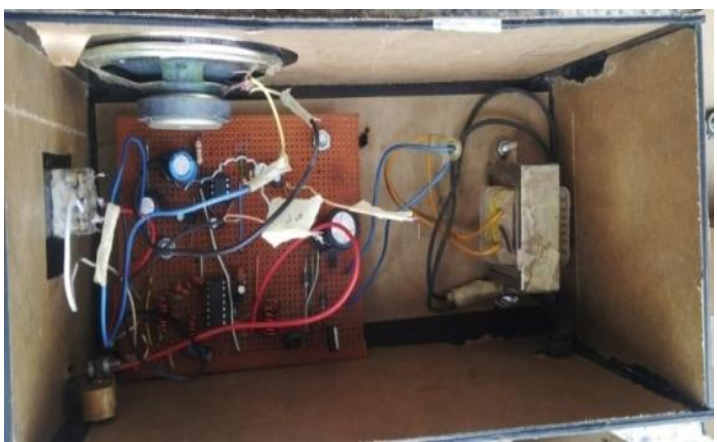

Plate 3.1: Receiver component of the audio surveillance system. 


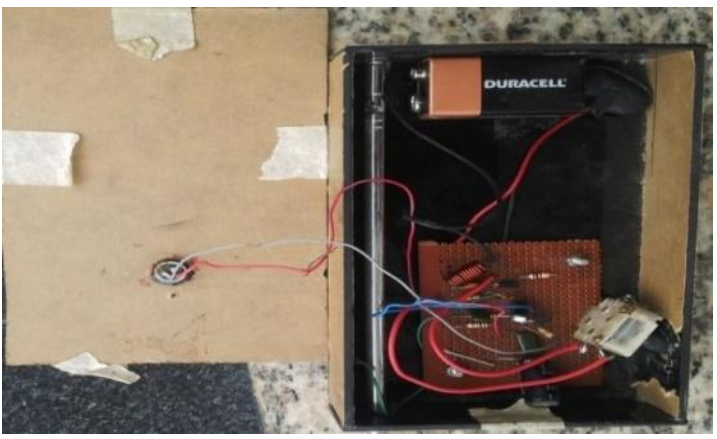

plate 3.2: Transmitter component of the audio surveillance system.

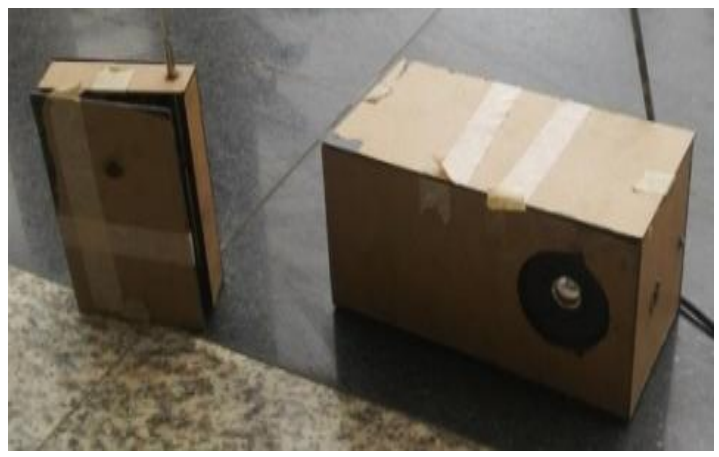

plate 3.3: Complete units of the system consisting of the Transmitter and the Receiver.

\section{DISCUSSION}

PROTEUS IDE was used to simulate the transmitter and notable result was recorded as shown in Fig 4 and 5. Also the same software was used on the Colpitt's oscillator that generated the result depict Fig 7. in a similar vein, after the construction, Fig. 8 shows the waveform of the input signal to the base of the base of the first transistor of the transmitter circuit when fed with audio signal and Fig. 9: The waveform of the input signal to the base of the second transistor of the transmitter circuit when fed with audio signal. The waveform of the output signal to the LC tank terminal of the transmitter circuit when fed with audio signal was depicted in Fig. 10 with Output of the receiver circuit (at speaker terminal) shown in Fig. 11 and Transmitted signal (oscillator output) in Fig. 12, Received signal in Fig. 13. The Fig. 3 depicts the AM/FM radio monolithic integrated circuit which is widely used for $\mathrm{AM} / \mathrm{FM}$ receivers with features such as $\mathrm{AM} \mathrm{HF}$ amplifier, local oscillator, mixer, IF amplifier, detector circuit, FM HF amplifier, local oscillator, mixer, IF amplifier, discriminator circuit, AGC circuit and the AM/FM band selection circuit was used to achieve the possible result. Fig. 14 shows waveform of the output signal from speaker terminal of the transmitter circuit when fed with audio signal has your transmitter circuit speaker terminals. The Fig. 15 through 17 shown the Receiver component of the audio surveillance, Transmitter component of the audio surveillance system and complete units of the system consisting of the Transmitter and the Receiver respectively.

\subsection{Conclusion}

In this research, an audio-based surveillance system was designed and implemented using CD2003 in place of discrete components. The performance of the system was significantly improved by replacing the discrete components with thelintegrated circuit in the design approach. The correlation muting system and the FLL (Frequency- LockedLoop) make it easy to tune and higher performance portables and clock radios, variable-capacitance, diode tuning and station presetting facilities were recorded.

\section{ACKNOWLEDGEMENT}

Our thanks to the experts who have contributed towards the development of the template.

\section{REFERENCES}

[1] Dempsey M. 2007. "Biometrics and the body as information: normative issues of the socio-technical coding of the body." Surveillance as Social Sorting: Privacy, Risk, and Automated Discrimination. Verified Identity Pass, Inc.

[2] O’Brien, T. 2009. Public Audio Surveilance Hits London.

[3] Peterson J. K. 2014. Understanding Surveillance Technologies: Spy Devices, CRC Press, Third Edition. ISBN: 084938320X

[4] Webster, W. R., Töpfer, E., Klauser, F. R., and Raab, C. D. 2011. Revisiting the surveillance camera revolution: Issues of governance and public policy. Introduction to part one of the Special issue. Information Polity. 16, 297-301.

[5] Agustina, J.R., Clavell, G.G. The impact of CCTV on fundamental right and crime prevention strategies: The case of the Catalan Control Commission of Video surveillance Devices. Computer law \& security review. 2011, 27, 168-74.

[6] Bai, Y. W., Xie, Z. L., Li, Z. H. 2011. Design and Implementation of a Home Embedded Surveillance System with Ultra-Low Alert Power. IEEE Transactions on Consumer Electronics.751539.

[7] Loomans, M. J. H., Koeleman, C. J. 2011. LowComplexity Wavelet-Based Scalable Image \& Video Coding for Home-Use Surveillance. IEEE Transactions on Consumer Electronics, 57, 50715.

[8] Rougier, C., Meunier, J., St-Arnaud, A., Rousseau, J. 2011. Robust Video Surveillance for Fall Detection Based on Human Shape Deformation. IEEE Transactions on Circuits and Systems for Video Technology, 21, 61122.

[9] Jeong, J., Gu, Y., He, T., Du, D. H. C. 2010. Virtual Scanning Algorithm for Road Network Surveillance. IEEE Transactions on Parallel and Distributed Systems. 21, 1734-49.

[10] Su, P. C., Wu, C. Y. 2010. A Joint Watermarking and ROI Coding Scheme for Annotating Traffic Surveillance Videos.EURASIP Journal on Advances in Signal Processing. Pp. 1-14.

[11] Sumalee, A., Zhong, R. X., Pan, T. L., Szeto, W. Y. 2011. Stochastic cell transmission model (SCTM): A stochastic dynamic traffic model for traffic state surveillance and assignment. Transportation Research Part B. 2011, 45, 507-5033. 
[12] Yuan J. L., Vehicle Surveillance with a Generic, Adaptive, 3D Vehicle Model. IEEE Transactions on Pattern Analysis and Machine Intelligence. 33, 1457-69.

[13] Luo, X., Wu, Y., Huang, Y., Zhang, J. 2011. Vehicle flow detection in real-time airborne traffic surveillance system. Transactions of the Institute of Measurement and Control/ 33, 880-97.

[14] Monperrus, M., Long, B., Champeau, J., Hoeltzener, B., Marchalot, G., Jézéquel, J. M. 2013. Model-Driven Architecture of a Maritime Systematic Review and Classification on Video Surveillance Systems 97. MECS I.J.

[15] Szpak, Z. L., Tapamo, J. R. 2011. Maritime surveillance: Tracking ships inside a dynamic background using a fast level-set. Expert Systems with Applications. 38, 666980.

[16] Conte, D., Foggia, P., Percannella, G., Tufano, F., and Vento, M. A. 2010. Method for Counting Moving People in Video Surveillance Videos.EURASIP Journal on Advances in Signal Processing, Pp. 1-10.

[17] Takahashi, M., Fujii, M., Shibata, M., Satoh, S. I. 2010. Robust Recognition of Specific Human Behaviors in Crowded Surveillance Video Sequences.EURASIP Journal on Advances in Signal Processing, Pp. 1-14.

[18] Amato, A., Lecce, V. D. 2011. Semantic Classification of Human Behaviors in Video Surveillance Systems. Journal WSEAS Transactions on Computers archive, 10, 343-52.

[19] Heilmann, E. 2011. Video surveillance and security policy in France: From regulation to Widespread acceptance. Information Polity, 16, 369-77.
[20] Räty, T. D. 2010. Survey on Contemporary Remote Surveillance Systems for Public Safety. IEEE Transactions on Systems, Man, and Cybernetics-Part C: Applications and Reviews, 40, 493-515.

[21] Paola, D. D., Milella, A., Cicirelli, G., Distante, A. 2010. An Autonomous Mobile Robotic System for Surveillance of Indoor Environments, International Journal of Advanced Robotic Systems, 7, 19-26.

[22] Zhang, J., Song, G., Qiao, G., Meng, T., Sun, H. 2011. An Indoor Security System with a Jumping Robot as the Surveillance Terminal, IEEE Transactions on Consumer Electronics.

[23] John W. K., 2011. Hierarchical and Modular Surveillance Systems in ITS IEEE Intelligent Systems, 26, 10-5.

[24] Bhavya, D. 2012. Super-heterodyne FM Receiver Design and Simulation University of Florida, Gainesville, FL, 32608, USA.

[25] Hongwu, T., and Fredrik, S. 2003. An RF Amplifier for a FM - Radio Receiver 88-108 MHz, Radio Project, Electroscience, Lund University.

[26] Areeb, A. 2011. Wireless USB-FM Transmitter using ROHM Semiconductor chip in VQFP-64 Packaging $\mathrm{PhD}$ fellow in Electronics \& Communication, Izmir Institute of Technology, Turkey MSc Electronics \& Communication, University of Teesside, UK 12/B.

[27] Chunbing, P. 1999. The Vision Machine, Trans. J. Rose. London: British Film Institute. 